\title{
COMPLETAMIENTO DE SERIES TEMPORALES DE NIVELES FREÁTICOS EN UN ÁREA DEPRIMIDA DE LA LLANURA BONAERENSE
}

\section{COMPLETATION OF TIME SERIES OF WATER TABLE LEVELS IN A FLATLAND AREA OF THE BUENOS AIRES PROVINCE}

\author{
Erik Zimmermann ${ }^{(1)(2)}$ \\ ${ }^{(1)}$ Facultad de Ciencias Exactas, Ingeniería y Agrimensura, Universidad Nacional de Rosario. Rosario. Santa Fe. Argentina \\ ${ }^{(2)}$ Consejo Nacional de Investigaciones Científicas y Técnicas. CONICET, Argentina. \\ e_mail: erikz@fceia.unr.edu.ar
}

\begin{abstract}
RESUMEN
Se presenta como objetivo el completamiento de registros temporales de niveles freáticos someros en un sector deprimido perteneciente a la cuenca del Arroyo del Azul (provincia de Buenos Aires), en el marco de una modelación matemática de la hidrología subterránea. Los registros completados conforman la condición de contorno aguas arriba del dominio de modelación. Se contemplaron diferentes series temporales para correlacionarlas con los niveles freáticos: a) anomalías acumuladas de precipitación, b) anomalías gravimétricas (misión satelital GRACE) y c) anomalías acumuladas hidrológicas (diferencia entre la precipitación y la evapotranspiración potencial). De los seis pozos freatimétricos seleccionados para el análisis y para el período donde se presentan las series en forma completa (oct 2014- abr 2016) se deduce que las anomalías de precipitación (a) presentan los coeficientes de correlación $r$ más bajos y fue descartada como serie análoga. Las anomalías gravimétricas mensuales (b), Gravity Recovery and Climate Experiment (GRACE) misión conjunta de la NASA y el Centro Aeroespacial Alemán (DLR) presentaron correlaciones buenas, con valores de $r$ entre 0.624 y 0.798, aunque la serie suministrada se discontinúa desde 2017. Las anomalías hidrológicas acumuladas, que fueron calculadas a escala diaria y retrasadas respecto los niveles un lag semanal para mejorar las regresiones, presentaron correlaciones muy buenas, con $r$ entre 0.861 y 0.894 , fueron las seleccionadas como serie análoga de completamiento. Considerando la mejor disponibilidad de datos climáticos frente a los satelitales y en virtud de los buenos resultados alcanzados se recomienda el uso de esta metodología en sectores de niveles freáticos someros donde el proceso de evaporación puede considerarse una componente hidrológica de importancia.
\end{abstract}

Palabras clave: completamiento de series temporales, anomalías gravimétricas, anomalías hidrológicas

\begin{abstract}
The objective is to complete temporary records of shallow water tables in a depressed sector belonging to the Azul river basin (province of Buenos Aires), within the framework of a groundwater mathematical modelling. The completed records make up the upstream boundary condition of the fluxes in the domain. Different time series were considered to correlate them with the water table levels: a) accumulated precipitation anomalies, b) gravimetric anomalies (GRACE satellite mission) and c) accumulated hydrological anomalies (difference between precipitation and potential evapotranspiration). From the six wells selected for the analysis and for the period where the series have completed records (Oct 2014-Apr 2016) it can be deduced that the precipitation anomalies (a) present the lowest correlation coefficients $r$ and it was discarded as analog series. The monthly gravimetric anomalies (b), Gravity Recovery and Climate Experiment (GRACE) joint mission of NASA and the German Aerospace Center (DLR) showed good correlations, with values of $r$ ranged between 0.624 and 0.798 , although the series support has been discontinued since 2017. The accumulated hydrological anomalies, which were calculated on a daily scale and lagged with respect to the water levels by a weekly lag to improve the regressions, presented very good correlations, with $r$ ranged between 0.861 and 0.894 , and were selected as an analog completion series. Considering the better availability of climatic data compared to satellite data and good results achieved, the use of this methodology is recommended in sectors with shallow groundwater levels where the evaporation process can be considered an important hydrological component.
\end{abstract}

Keywords: completion of time series, gravimetric anomalies, hydrological anomalies 


\section{INTRODUCCIÓN}

El trabajo se plantea en el marco de una modelación matemática de la hidrología subterránea con el fin de conceptualizar los principales procesos hidrológicos que se verifican en la zona de estudio propuesta.

El área de estudio se focaliza en la cuenca del arroyo del Azul, se ubica en la zona central de la provincia de Buenos Aires, entre los $58^{\circ} 51^{\prime}$ y $60^{\circ} 10^{\prime}$ de longitud oeste y $36^{\circ} 09^{\prime}$ y $37^{\circ} 19^{\prime}$ de latitud sur (Figura 1).

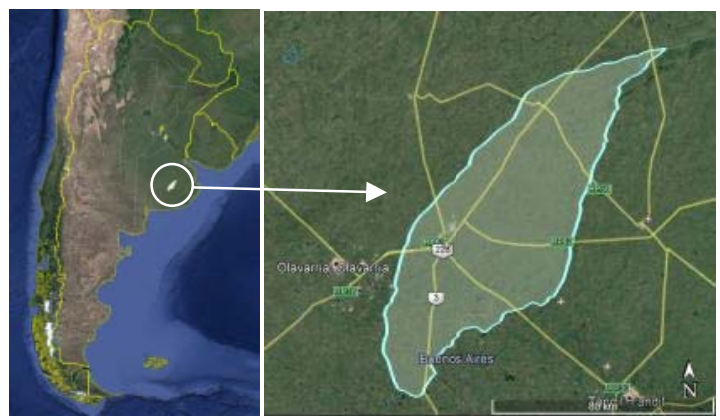

Figura 1. Cuenca del arroyo del Azul.

La cuenca abarca una superficie de $6237 \mathrm{~km}^{2}$ y como formas destacables se reconocen un subambiente serrano hacia el sur de la cuenca con altitudes por sobre los $200 \mathrm{msnm}$ (pendiente media del terreno del $5 \%$ ), y un sub-ambiente de llanura hacia el norte, por debajo de los $130 \mathrm{msnm}$ (con pendientes que varían entre 0.5 y $0.1 \%$ ), con una zona de transición entre ambos, caracterizada por suaves ondulaciones (Fajardo y Entraigas, 2017).

En el sector más bajo, sumamente llano, con sus suelos nátricos y drenaje deficiente, se desarrolla principalmente la cría ganadera extensiva en una matriz de pastizales naturales interrumpida por las numerosas cubetas y lagunas de carácter mayoritariamente semi-permanente $\mathrm{y}$ por dunas parabólicas y longitudinales.

Desde el punto de vista hidrológico, lo más notable del comportamiento del agua en cuencas tan deprimidas como la del Azul es la acumulación del agua sobre la superficie, y la interacción que se establece entre las aguas superficiales y las subterráneas, conformando un Sistema Hidrológico No Típico.

Específicamente se ha concentrado el interés en la modelación de los sectores más deprimidos de la cuenca (Figura 2).

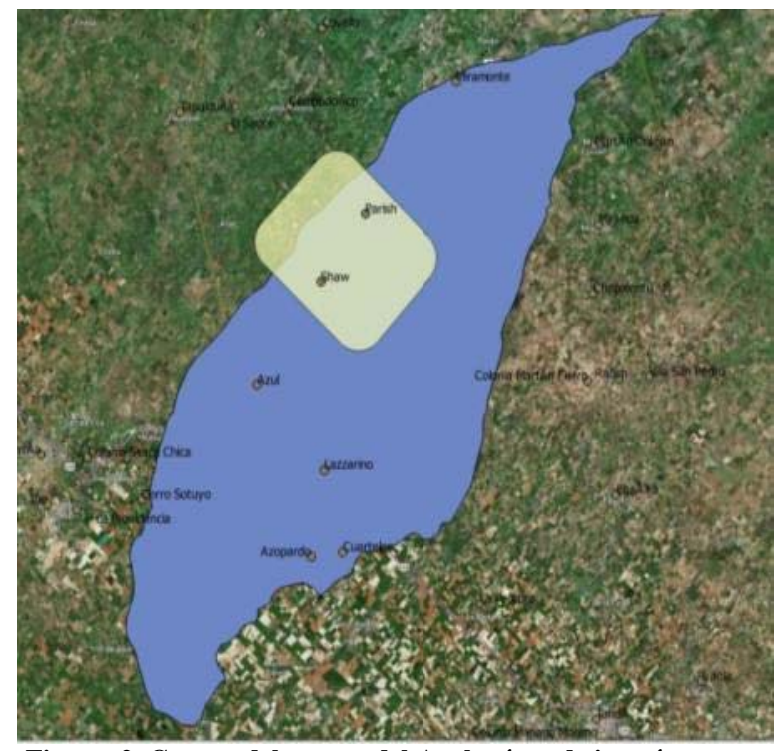

Figura 2. Cuenca del arroyo del Azul y área de interés para la modelación.

\section{CONDICIONES DE CONTORNO DE LA MODELACIÓN}

El dominio de modelación consistió en un rectángulo de $24 \mathrm{~km}$ en dirección SO-NE y $29 \mathrm{~km}$ en dirección NO-SE, el que se encuentra en su mayor parte incluido en la cuenca superficial del arroyo del Azul (Figura 2), que incluyen los pozos de observación de niveles freáticos y se encuentra intersectada por la ruta nacional $\mathrm{N}^{\circ} 3$. La grilla de discretización fue subdividida en elementos cuadrados de $1 \mathrm{~km}$ de lado (Figura 3), totalizando 696 celdas (24 filas, 29 columnas).

El dominio de modelación se encuentra en un sector de interfluvio de cuencas superficiales por lo que resultó dificultoso establecer condiciones de borde. Las isofreáticas construidas para distintos momentos de mediciones sí manifestaron un patrón de flujo repetitivo, orientado hacia el $\mathrm{NE}$, lo que permitió establecer hipótesis de contornos de no flujo en los laterales. En los contornos aguas arriba y aguas abajo se propusieron condiciones de niveles y flujos preestablecidos, respectivamente (Figura 3). La elección de las condiciones de contorno de los bordes SO y NE surgió en función de la información disponible. El borde SO cuenta con registros freatimétricos históricos en muchos pozos de ese frente. Complementariamente se consideró la condición de control de flujos regionales en el borde opuesto (NE) hacia el río Salado. 


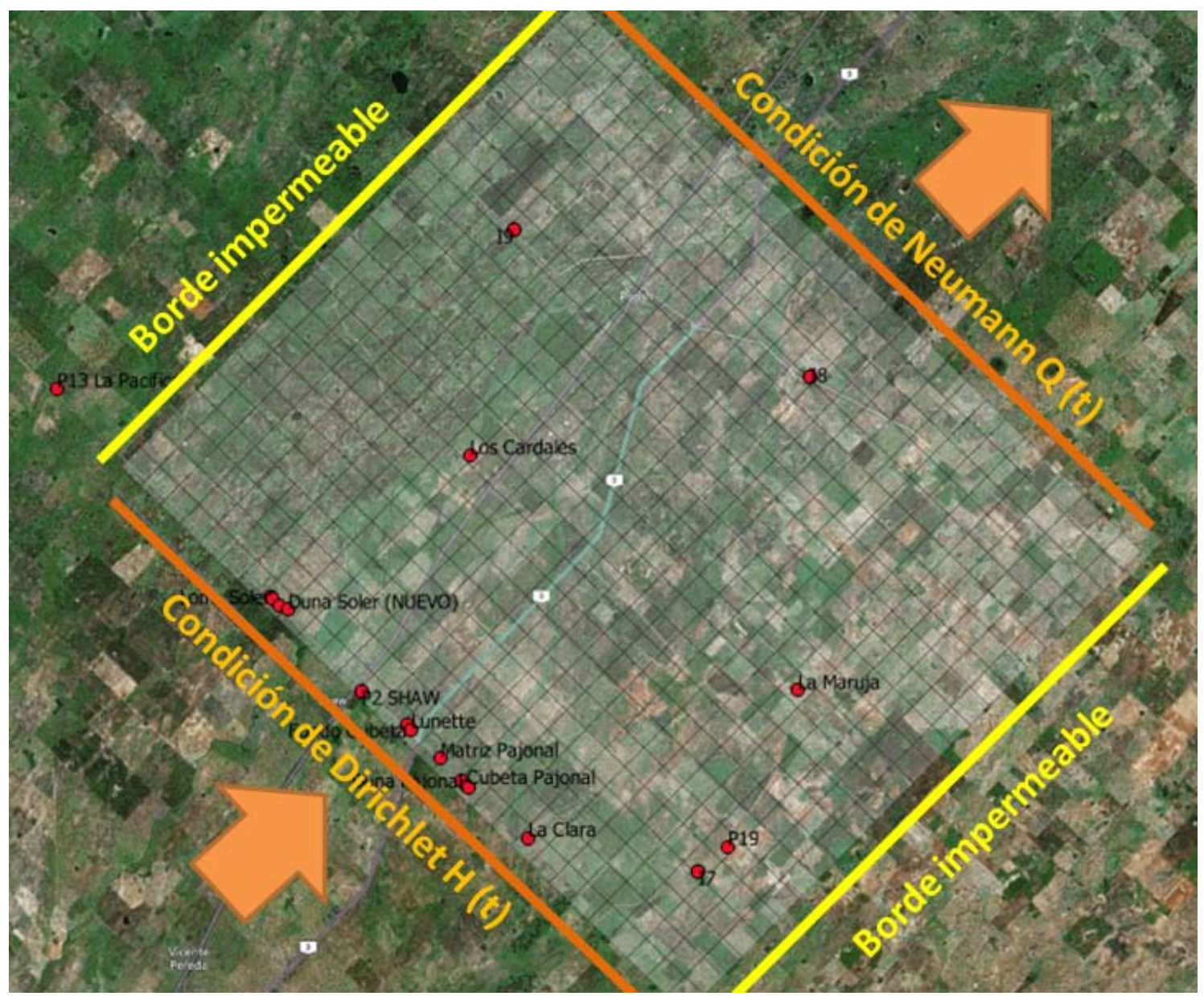

Figura 3. Grilla de modelación propuesta, pozos de observación de niveles freáticos y condiciones de borde.

\section{PROCESAMIENTO DE LOS NIVELES FREÁTICOS AGUAS ARRIBA}

Los registros históricos de niveles freáticos en el frente SO fueron discontinuos temporalmente $\mathrm{y}$ abarcan pocas celdas representativas. Son nueve pozos en los $29 \mathrm{~km}$ del frente con registros mensuales desde octubre de 2014 hasta mediados de 2018, con prácticamente un año (abril 2016 a marzo 2017) de discontinuidad.

El período de simulación del modelo se propuso en función de la información disponible entre octubre de 2014 a abril de 2018, en total 42 meses.

En principio, dada la importancia de contar con un registro continuo de niveles en la condición de borde, se propuso completar la serie de registros discontinuos mediante correlaciones con patrones análogos, y tras una selección previa, se consideraron las anomalías de precipitación y de gravedad (misión GRACE).
Las anomalías de precipitación se refieren a las diferencias de lluvia mensual respecto a la media del período analizado, posteriormente en forma acumulada.

Respecto a las anomalías de gravedad, la Gravity Recovery and Climate Experiment (GRACE) fue una misión conjunta de la NASA y el Centro Aeroespacial Alemán (DLR). Los satélites gemelos tomaron medidas detalladas de las anomalías del campo gravitatorio de la Tierra desde su lanzamiento en marzo de 2002 hasta el final de su misión científica en octubre de 2017. Al medir las anomalías de la gravedad, GRACE mostró cómo la masa se distribuye alrededor del planeta y cómo varía con el tiempo. Los productos del satélite estiman cambios en la masa en unidades de espesor equivalente de agua líquida. Si bien las mediciones están afectadas por grandes masas de agua se analizó la posibilidad de correlacionar las anomalías de gravedad con los niveles freáticos observados en el sector de estudio. 
El análisis se correlaciones se realizó en 9 pozos del frente SO (camino a la vieja estación de FFCC Shaw), representativos de 4 celdas de modelación (674, 681, 682, 684 - Figura 4).

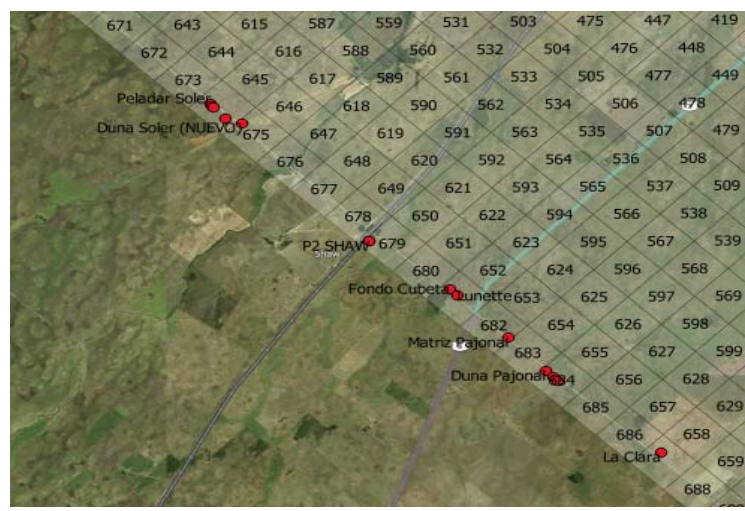

Figura 4. Pozos freatimétricos y celdas de modelación

Para construir las anomalías de precipitación, se contó con datos diarios de las estaciones Azul (Aeródromo) y la estación SHAW (ex FFCC). La primera distante unos $27 \mathrm{~km}$ del sector pero considerada estación de confianza y la segunda ubicada prácticamente en el sector. Se contó con información de evapotranspiración estimada y con un balance hidrológico en el área de La Baguala (Varni y Custodio, 2013; Vercelli et al., 2019), ubicada en el interior del dominio de modelación.
El planteo inicial consistió en la estimación de anomalías de precipitación con información agregada mensualmente, considerando algunos registros de pozos y su contraste gráfico.

Las profundidades freáticas en el área se dan someramente (en algunos pozos alrededor del metro o menos), lo que pone de manifiesto que la evapotranspiración constituiría una componente de importancia, por lo que se analizaron anomalías de las diferencias entre precipitación (P) y evapotranspiración potencial (ETo), denominadas anomalías hidrológicas. Los valores de ETo fueron estimados mediante método de Penman-Monteith por Varni y Custodio (2013). Como se observa en la Figura 5 las anomalías hidrológicas reflejan mucho mejor los patrones temporales de niveles freáticos.

En términos de coeficiente de correlación las anomalías de $\mathrm{P}$ presentaron un $r$ promedio de 0.14 mientras que las de P-ETo un $r$ medio de 0.58 en la totalidad de los pozos considerados para el período completo. Si se analiza el último período continuo de datos (marzo 2017 - abril 2018) el coeficiente de correlación promedio para la totalidad de los pozos alcanza del valor de 0.82 para las anomalías hidrológicas. Por esta razón se consideraron solamente éstas últimas anomalías descartando las de precipitación.

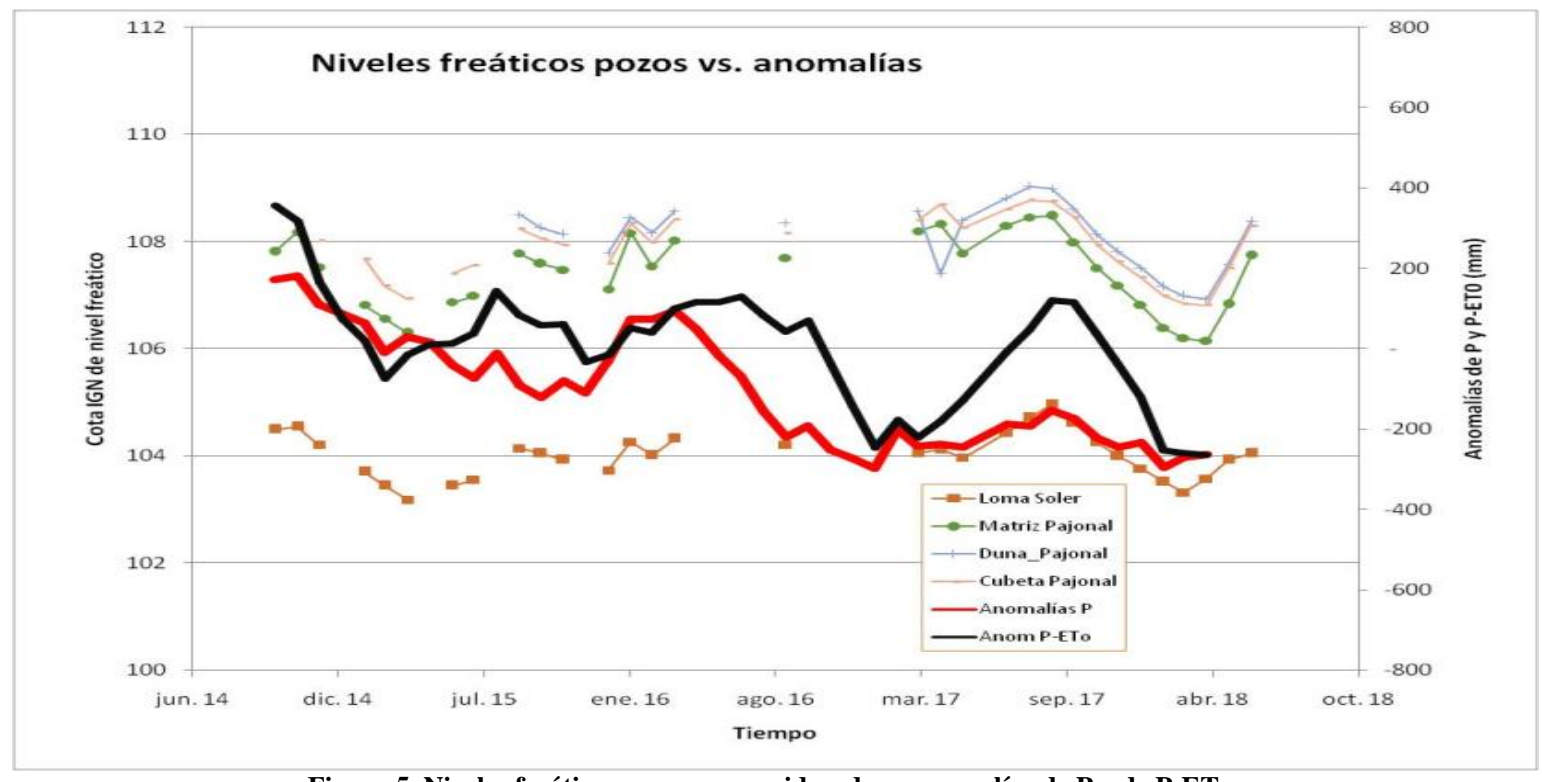

Figura 5. Niveles freáticos en pozos considerados y anomalías de P y de P-ETo 
Paralelamente se obtuvieron los valores de las anomalías gravimétricas que están disponibles en períodos mensuales. Se extractaron datos desde octubre de 2014 a enero de 2017 (28 meses). Para el análisis de las correlaciones se consideraron 15 meses en donde la información de las anomalías gravimétricas e hidrológicas (P-ETo) cuentan con información de freatrimetría que van desde octubre de 2014 a abril de 2016 (con 4 meses faltantes).

Las anomalías hidrológicas se estimaron también en forma diaria, desplazando las series hasta unas tres semanas, es decir, lags entre 0 y19 días, previendo un retardo en la reacción de los niveles respecto a las variables climáticas, analizando las correlaciones entre las series. En la Figura 6 puede observarse que los máximos $r$ se observan para lags 7 días y 12 días, siendo mayores en el conjunto para el retardo de una semana.

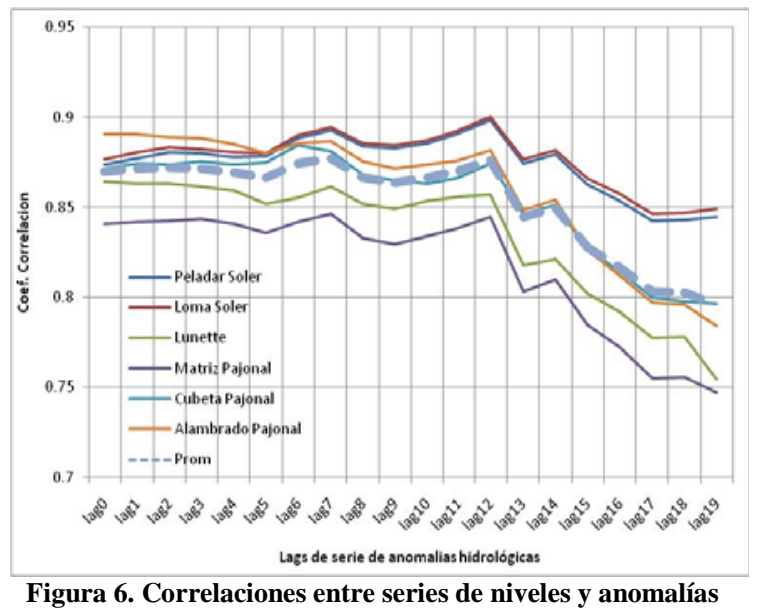
hidrológicas desplazadas temporalmente.

De los 9 pozos con información freatimétrica del camino Shaw se seleccionaron 6 que son los que tienen menor interrupción de registros. La Tabla 1 muestra los coeficientes de correlación para cada metodología. Las anomalías hidrológicas se consideraron desplazadas en una semana.
Adicionalmente se consideró una regresión múltiple con ambas anomalías. Puede observarse que no aportó demasiada ventaja la regresión múltiple. Posteriormente se detectó que existe una correlación importante entre ambas anomalías ( $r=0.793$, Figura 7).Los ajustes alcanzados por las metodologías mencionadas se muestran en la Figura 8.

Tabla 1. Coeficientes de correlación entre anomalías y niveles freáticos de los pozos seleccionados

\begin{tabular}{|c|c|c|c|}
\hline $\begin{array}{c}\text { Anomalías } \\
\text { Pozos }\end{array}$ & Hidrológicas & Gravimétricas & Ambas \\
\hline $\begin{array}{c}\text { Peladar } \\
\text { Soler }\end{array}$ & 0.893 & 0.794 & 0.905 \\
\hline $\begin{array}{c}\text { Loma } \\
\text { Soler }\end{array}$ & 0.894 & 0.798 & 0.909 \\
\hline Lunette & 0.861 & 0.624 & 0.834 \\
\hline $\begin{array}{c}\text { Matriz } \\
\text { Pajonal }\end{array}$ & 0.846 & 0.668 & 0.835 \\
\hline $\begin{array}{c}\text { Cubeta } \\
\text { Pajonal }\end{array}$ & 0.881 & 0.748 & 0.885 \\
\hline $\begin{array}{c}\text { Alambrado } \\
\text { Pajonal }\end{array}$ & 0.887 & 0.702 & 0.880 \\
\hline
\end{tabular}

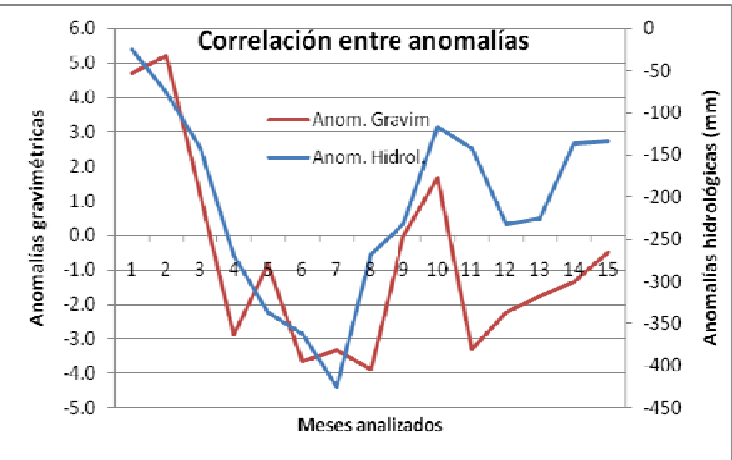

Figura7.Correlación entre anomalías gravimétricas e hidrológicas

Considerando que la correlación con las anomalías hidrológicas es mayor y además representa un modelo simple y fácil de construir con datos climáticos (generalmente accesibles) se optó por considerarla como metodología de completamiento temporal de los registros freatimétricos. 

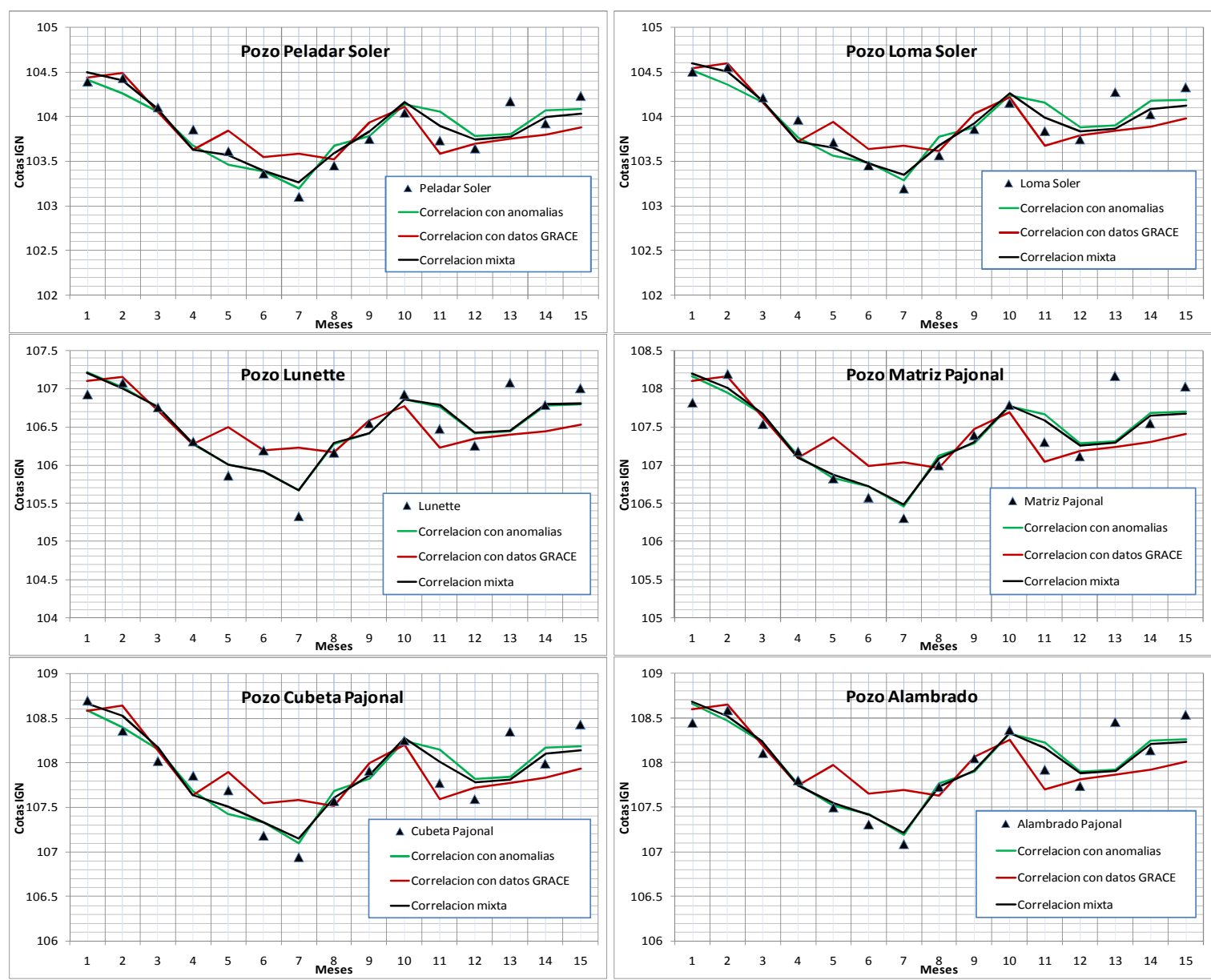

Figura 8. Ajustes entre pronósticos de niveles freáticos mediante anomalías hidrológicas (línea continua verde), anomalías gravitacionales (línea continua roja), ambas anomalías (línea continua negra) y niveles registrados (triángulos negros)

\section{CONCLUSIONES}

Se presenta como objetivo el completamiento de registros temporales de niveles freáticos someros en un sector deprimido perteneciente a la cuenca del Arroyo del Azul, provincia de Buenos Aires. Para ello, se han contemplado diferentes series temporales para correlacionarlas con los niveles: a) anomalías acumuladas de precipitación, b) anomalías gravimétricas (misión satelital GRACE) y c) anomalías acumuladas hidrológicas (diferencia entre la precipitación y la evapotranspiración potencial). De los seis pozos freatimétricos seleccionados para el análisis y para el período donde se presentan las series en forma completa (oct 2014- abr 2016) se deduce que las anomalías de precipitación (a) presentan los coeficientes de correlación $r$ más bajos y fue descartada como serie análoga. Las anomalías gravimétricas (b) presentaron correlaciones buenas, aunque la serie suministrada se discontinúa desde
2017. Las anomalías hidrológicas acumuladas, que fueron estimadas a escala diaria y regresadas linealmente con los niveles mediante un retardo semanal, presentaron correlaciones muy buenas y fueron las seleccionadas como serie análoga de completamiento. Considerando la mejor disponibilidad de datos climáticos frente a los satelitales y en virtud de los buenos resultados alcanzados se recomienda el uso de esta metodología en sectores de niveles freáticos someros donde el proceso de evaporación puede considerarse una componente hidrológica de importancia.

\section{REFERENCIAS}

Fajardo L. y Entraigas, I. (2017). Conectividad hidrológica en un sistema hidrológico no típico. Caso del sector llano de la cuenca del Arroyo del Azul. Informe de avance beca doctoral CIC. 
IHLLA. Azul. Argentina.

Varni, M. y Custodio, E. (2013). Evaluación de la recarga al acuífero del Azul: 1. Análisis de las características climáticas. VIII Congreso Hidrogeológico Argentino y VI Seminario Hispano Latinoamericano sobre temas actuales de hidrología subterránea. La Plata. Argentina.

Vercelli, N.; Varni, M; Lara, B.; Entraigas, I. y Ares, G. (2019). Linking soil water balance with flood spatial arrangement in an extremely flat landscape. Hydrological Processes 34(1), 21-32. DOI: 10.1002/hyp.13567.
Tipo de Publicación: NOTA TÉCNICA.

Trabajo recibido el 02/09/2020 y aprobado para su publicación el 06/10/2020.

\section{COMO CITAR}

Zimmermann, E. (2020) Completamiento de series temporales de niveles freáticos en un área deprimida de la llanura bonaerense. Cuadernos del CURIHAM. 26:55-61. DOI: https://doi.org/10.35305/curiham.v26i0.148

Este es un artículo de acceso abierto bajo licencia: Creative Commons Atribución - No Comercial Compartir Igual 4.0 Internacional (CC BY-NC-SA 4.0) (https://creativecommons.org/licenses/by-ncsa/4.0/deed.es) 